\title{
Mixed-Reality-in-the-Loop Simulation zur Schulung technischer Fachkräfte im Maschinen- und Anlagenbau
}

\author{
Jana Hönig ${ }^{1}$, Marc Schnierle ${ }^{1}$, Camilla Wehnert ${ }^{2}$, Daniel Littfinski ${ }^{3}$, Christian \\ Scheifele $^{4}$, Denis Pfeifer ${ }^{4}$, Carlos Münster ${ }^{5}$, Armin Roth ${ }^{5}$, Julia Franz ${ }^{2}$, Sascha \\ Röck ${ }^{1}$, Alexander Verl $^{3}$
}

\section{Zusammenfassung}

Dieser Beitrag stellt die Mixed-Reality-in-the-Loop Simulation (MRiLS) zur Schulung technischer Fachkräfte im Maschinen- und Anlagenbau vor. Die MRiLS koppelt die aus dem Engineering bereits vorhandenen Modelle der Hardware-in-the-Loop Simulation (HiLS) mit Visualisierungs- und Interaktionsmethoden der Mixed Reality (MR) und integriert dadurch den Nutzenden und dessen Verhalten sowie die reale Umgebung vollständig in den Simulationskreislauf. Der Beitrag thematisiert neben der notwendigen Middleware zur Kopplung der HiLS mit der MR-Umgebung auch die Steuerungsbelastung durch Multiuser-Zugriffe. Die Funktionsfähigkeit des vorgestellten Konzepts wird anhand eines ausgewählten beispielhaften Automatisierungssystems belegt. Für das Automatisierungssystem wird der Aufbau der MRiLS sowie das Konzept für den Ablauf einer Schulung mittels MRiLS vorgestellt.

\section{Stichwörter}

Mixed-Reality-in-the-Loop Simulation, Digitaler Zwilling, Mixed Reality, Augmented Reality, Virtual Reality, Hardware-in-the-Loop Simulation, Schulung, Maschinen- und Anlagenbau

\section{Formen der Wissensvermittlung im Maschinen- und Anlagenbau}

Der Maschinen- und Anlagenbau ist durch steigende Automatisierungsgrade, komplexe Prozessabläufe und eine zunehmende Vernetzung von Maschinen und Anlagen charakterisiert. Durch die somit wachsende Komplexität sowie vor dem Hintergrund des Fachkräftemangels kommt der Aus- und Weiterbildung technischer Fachkräfte eine zentrale Rolle zu.

Schulungen im Maschinen- und Anlagenbau erfolgen bislang vorwiegend an der realen Anlage und werden bei ausgewählten Lernszenarien um virtuelle Lerngegenstände (z.B. Webbased Trainings, Remote-Schulungen oder PC-basierte Schulungen vor Ort) ergänzt (siehe Bild 1).

\footnotetext{
1 Virtual Automation Lab, Fakultät Maschinen und Systeme, Hochschule Esslingen

2 Professur für Erwachsenenbildung und Weiterbildung, Otto-Friedrich-Universität Bamberg

3 Institut für Steuerungstechnik der Werkzeugmaschinen und Fertigungseinrichtungen, Universität Stuttgart

$4 \quad$ ISG Industrielle Steuerungstechnik GmbH

$5 \quad$ Roth Steuerungstechnik GmbH
} 


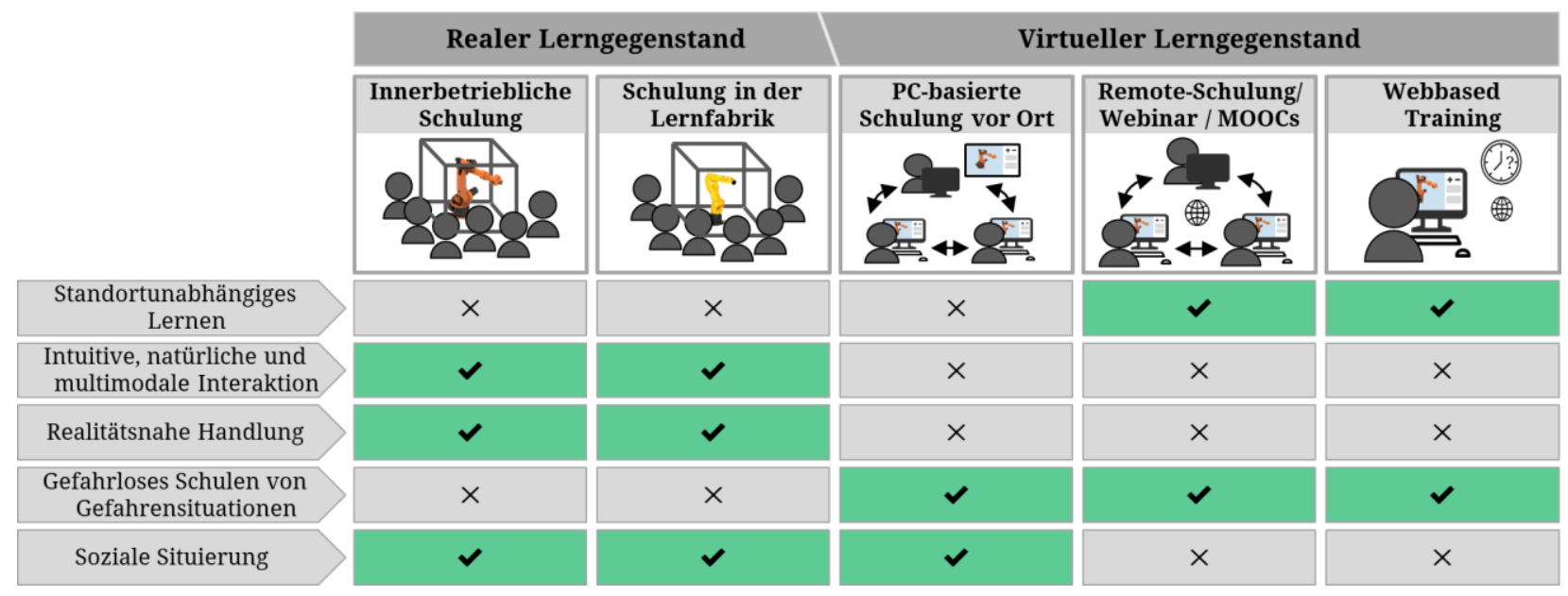

Bild 1: Methoden und Formen der Wissensvermittlung im Maschinen- und Anlagenbau (vgl. [1])

Die bislang eingesetzten Formen der Wissensvermittlung sind insbesondere im Hinblick auf die Interaktion zwischen Mensch und Maschine stark limitiert sowie beim Lernen an der realen Anlage mit Hemmnissen, Gefahrenpotenzialen und einer Standortgebundenheit verbunden. Standortunabhängige Methoden (z.B. Webbased Training) sind hingegen durch eine fehlende soziale Situierung sowie eine mangelnde Realitätsnähe der Handlungen geprägt.

Moderne Mixed Reality Technologien können an diesen Defiziten ansetzen und durch eine egozentrische standortunabhängige Visualisierung sowie eine multimodale Interaktion vielfältige Potenziale für neue Schulungsformate ermöglichen. Milgram et al. [2] beschreiben die Mixed Reality (MR) als stufenlose Kombination von virtuellen und realen Komponenten in einem gemeinsamen RealitätsVirtualitäts-Kontinuum. Entsprechend dem Verhältnis von realen und virtuellen Inhalten wird dieses Kontinuum in vier Kategorien unterteilt. Während die reale Umgebung (RE) ausschließlich physisch existierende Komponenten enthält, ist die Virtual Reality (VR) komplett computergeneriert. Zwischen diesen beiden Extremformen gibt es gemischte Umgebungen, die sowohl reale als auch virtuelle Komponenten enthalten. Eine Augmented Reality (AR) Umgebung umfasst mehr reale als virtuelle Inhalte, während bei der Augmented Virtuality (AV) die Relation umgekehrt ist.

Bisherige Implementierungen für Schulungen in Mixed Reality (z.B. [3], [4]) bilden das Anlagenverhalten zumeist ohne Integration industrieller Steuerungstechnik zeitaufwändig nach, wobei durch die notwendige Abstraktion des Verhaltens eine geringe Modell- und Schulungstiefe resultiert.

Im Entwicklungsprozess des Maschinen- und Anlagenbaus entstehen demgegenüber bei den steuerungsgekoppelten Hardware-in-the-Loop Simulationsmethoden (HiLS) Maschinenmodelle mit hoher Modelltiefe (vgl. [3]). Während die HiLS durch die Kopplung einer Steuerungsausprägung mit virtuellen Anlagenkomponenten (Digitaler Zwilling) zwar eine hohe Modelltiefe ermöglicht, ist die Integration des Menschen in den Simulationskreislauf durch die exozentrische Visualisierung auf zweidimensionalen Computer-Monitoren sowie die Interaktion über Maus und Tastatur weiterhin stark limitiert.

\section{Mixed-Reality-in-the-Loop Simulation (MRiLS)}

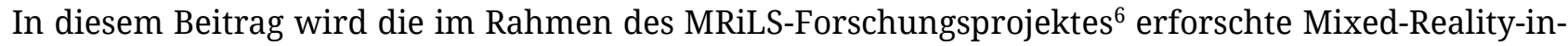
the-Loop Simulation (MRiLS) für die innerbetriebliche Schulung im Maschinen- und Anlagenbau vorgestellt.

6 Das Verbundprojekt „Hybrides Interaktionskonzept für Schulungen mittels Mixed-Reality-in-theLoop Simulation“ (Förderung durch Bundesministerium für Bildung und Forschung, 


\subsection{Allgemeiner Aufbau}

Die Mixed-Reality-in-the-Loop Simulation (MRiLS) baut auf der HiLS auf und integriert den Nutzenden und dessen Verhalten sowie die reale Umgebung durch die Kopplung der aus dem Engineering bereits bestehenden HiLS-Modelle mit Visualisierungs- und Interaktionsmethoden der Mixed Reality vollständig in die Simulationsumgebung (siehe Bild 2). Die MRiLS ermöglicht eine natürliche und an den menschlichen Sinnen orientierte Interaktion zwischen Nutzendem und Digitalem Zwilling, während die Integration der industriellen Steuerungstechnik das realistische Anlagenverhalten absichert.
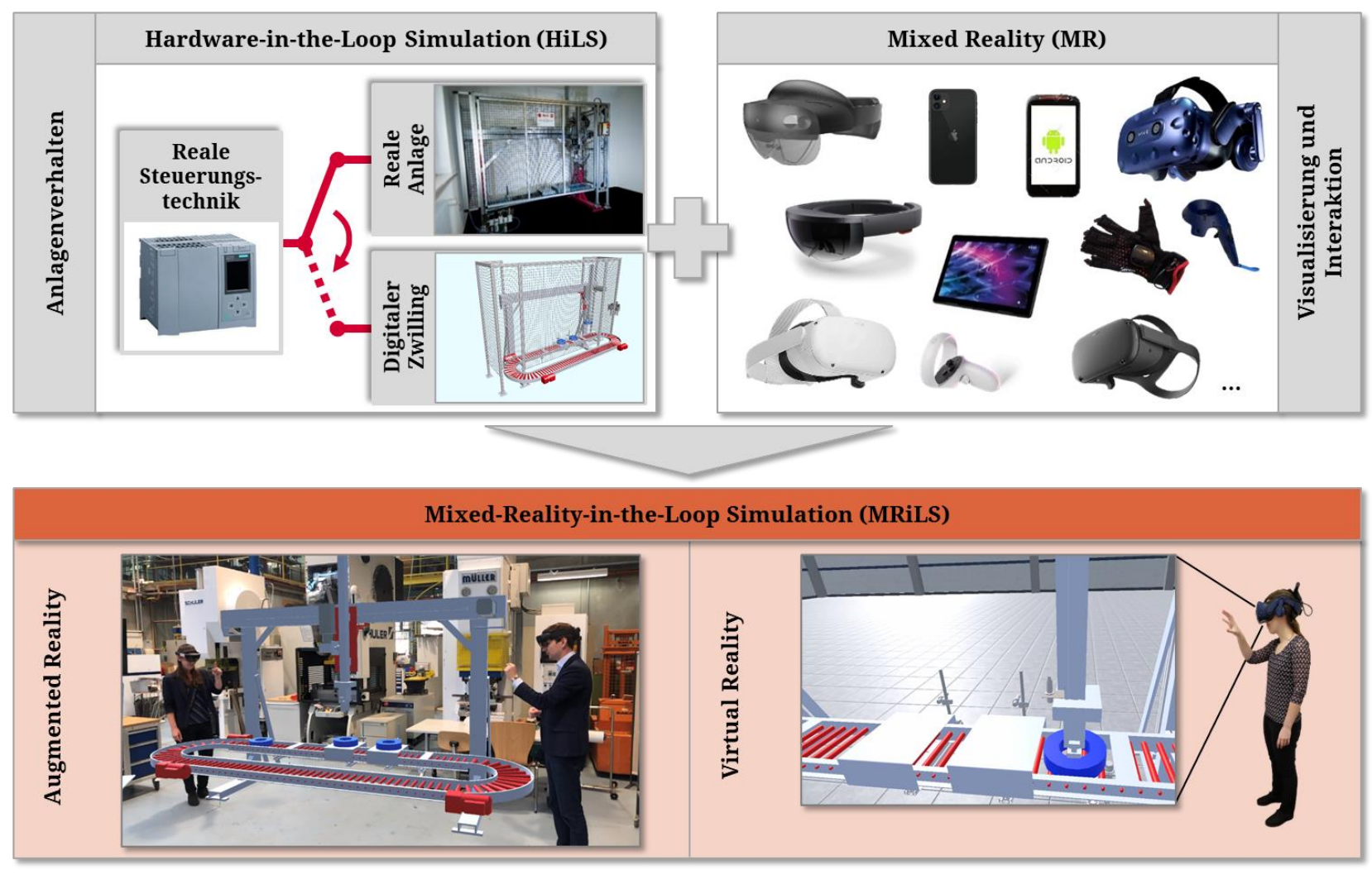

Bild 2: Aufbau der Mixed-Reality-in-the-Loop Simulation (MRiLS)

Ein zentrales Kriterium für die vollständige Integration des Menschen in den HiLS-Kreislauf und somit für die Schulung technischer Fachkräfte mittels MRiLS ist die Immersionsfähigkeit des Systems sowie das daraus resultierende subjektive Präsenzgefühl des Nutzenden. Das Präsenzgefühl beschreibt das (psychologische) Gefühl des Nutzenden, sich tatsächlich innerhalb der bereitgestellten mediatisierten Welt zu befinden (vgl. [6], [7]). Daraus ergeben sich folgende Leitziele für die MRiLS (vgl. [8]):

- Egozentrische Visualisierung: Die Bereitstellung der virtuellen Komponenten erfolgt in egozentrischer (vom Benutzer abhängiger) Perspektive, so dass der Nutzende diese dreidimensional im Raum betrachten kann.

Fkz.:16SV8344 - 16SV8348) wird von den industriellen Projektpartnern ISG Industrielle Steuerungstechnik $\mathrm{GmbH}$ und Ingenieurbüro Roth $\mathrm{GmbH} \& \mathrm{Co}$. KG mit den wissenschaftlichen Partnern Otto-Friedrich-Universität Bamberg, Professur für Erwachsenenbildung und Weiterbildung, Universität Stuttgart, Institut für Steuerungstechnik der Werkzeugmaschinen und Fertigungseinrichtungen (ISW) und Hochschule Esslingen, Virtual Automation Lab (VAL) durchgeführt. Die gewerblichen beruflichen Schulen des Landkreises Esslingen Max-Eyth-Schule Kirchheim, Friedrich-Ebert-Schule Esslingen sowie die Philipp-Matthäus-Hahn Schule Nürtingen sind als assoziierte Partner involviert. 
- Intuitive multimodale Mensch-Modell-Interaktion: Der Nutzende kann intuitiv und multimodal mit den virtuellen Komponenten interagieren und erhält eine umfassende Rückmeldung durch Erzeugung synthetischer Stimuli für alle relevanten Sinne (z.B. visuell, haptisch, auditiv).

- Kopplung realer und virtueller Komponenten: Die realen und virtuellen Anlagenkomponenten werden in einer gemeinsamen Umgebung visualisiert und beeinflussen sich gegenseitig, so dass das Realitäts-Virtualitätskontinuum vollständig umfasst wird.

- Multiuser-Kollaboration: Mehrere Nutzende können mit den virtuellen Komponenten kollaborativ in einer gemeinsamen Umgebung standortunabhängig interagieren und in dieser Umgebung intuitiv miteinander kommunizieren.

\subsection{Multiuser und Steuerungsbelastung}

Die Multiuser-Kollaboration erfordert neben der Kommunikation zwischen mehreren Schulungsteilnehmenden untereinander auch die Kommunikation zwischen den unterschiedlichen Schulungsteilnehmenden und der angebundenen Hardware-in-the-Loop Simulation zum Austausch der Zustandsund Interaktionsdaten. Die Zustandsdaten der HiLS sowie der daran angekoppelten Steuerungstechnik müssen dabei an alle Schulungsteilnehmenden verteilt werden, wodurch im Vergleich zu einem Single-Userzugriff mit steigender Teilnehmendenzahl der Kommunikationsaufwand sowie die Auslastung der Steuerung steigt. Zeitgleich müssen auch die Interaktionsdaten (z.B. Manipulation der Position einer virtuellen Komponente) der einzelnen Nutzenden an die HiLS übermittelt und entsprechend ausgewertet werden.

Der wachsende Kommunikationsaufwand bei steigender Anzahl der Nutzenden limitiert somit bei einer direkten Anbindung der Endgeräte an die HiLS die Skalierbarkeit der Teilnehmendenzahl beziehungsweise die möglichen Kommunikationstakte zwischen HiLS und Nutzenden. Eine Reduktion des Kommunikationstaktes kann bei dem Nutzenden zu einer wahrnehmbaren Verzögerung beim Verhalten des Digitalen Zwillings (z.B. bei Interaktionen) führen. Diese wahrnehmbaren Verzögerungen können eine fehlende Plausibilitätsillusion (z.B. Ausführung einer Manipulation eines virtuellen Objektes führt nicht bzw. zu einer verspäteten Manipulation) hervorrufen, wodurch ein Bruch im Präsenzgefühl entstehen kann.

Als Lösung für die Steuerungsbelastung durch die Multiuser-Kollaboration wird für die MRiLS eine Middleware zur Kopplung der HiLS-Modelle mit den Mixed Reality-Endgeräten vorgeschlagen (vgl. [9]). Bild 3 zeigt das Konzept der Middleware, die zugleich der Kopplung der Nicht-Echtzeit-Umgebung der Mixed Reality-Endgeräte mit der Echtzeit-Umgebung der HiLS dient.

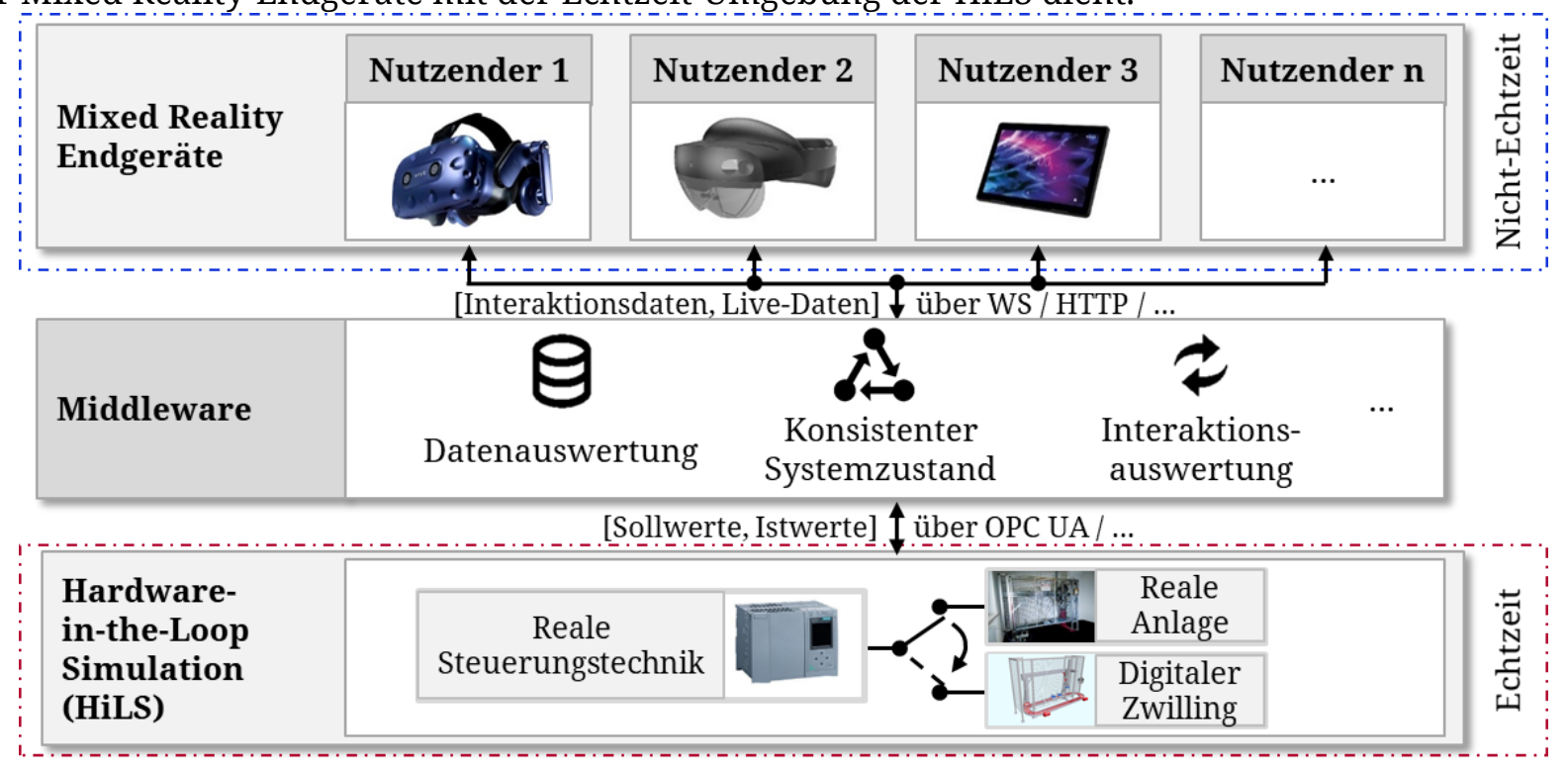

Bild 3: Middleware zur Kopplung von Mixed Reality Endgeräten und Hardware-in-the-Loop Simulationen 
Die Nutzenden koppeln sich über das jeweilige Endgerät (z.B. Augmented Reality-Brille, Virtual Reality-Brille, Tablet, etc.) an die Middleware und tauschen über gängige Technologien aus den Bereichen Web und Cloud (z.B. WS, HTTP) Live-Daten der MRiLS sowie die auf dem Endgerät bereits abstrahierten Interaktionsdaten der Nutzenden (siehe auch [10]) mit der Middleware aus. Die Instanzen der HiLS verbinden sich über industrielle Kommunikationsprotokolle (z.B. OPC UA) ebenfalls mit der Middleware und erhalten die Sollwerte des Digitalen Zwillings, generieren daraufhin Istwerte und schreiben diese an die Middleware zurück. In der Middleware erfolgt die Auswertung der abstrahierten Interaktionsdaten der angebundenen Endgeräte, die Auslösung entsprechender Events sowie die Bereitstellung eines konsistenten Systemzustandes für alle Nutzenden auf Basis der eingehenden Daten (z.B. Istwerte der HiLS).

Durch die Integration einer Middleware erfolgt die Kommunikation der Endgeräte und der HiLS nicht mehr direkt, sondern die Daten werden gebündelt über die Middleware an die angebundenen Instanzen verteilt. Dies hat zur Folge, dass die Kommunikationslast im Vergleich zu einer direkten Verbindung sinkt und die Belastung der HiLS sowie der angekoppelten Steuerungstechnik bei gleichbleibender Anzahl der Nutzenden sinkt. Durch den Einsatz der Middleware sind damit eine höhere Anzahl an Teilnehmenden sowie ein höherer Kommunikationstakt möglich. Die Middleware ermöglicht zudem die Homogenisierung der Kommunikation zwischen Endgeräten und der HiLS, so dass nicht auf jedem Endgerät selbst das industrielle Kommunikationsprotokoll implementiert werden muss.

\subsection{Potenziale und Mehrwerte}

Der Einsatz der MRiLS bietet zahlreiche Potenziale für die Schulung technischer Fachkräfte im Maschinen- und Anlagenbau:

- Präsenzgefühl des Nutzenden im Simulationskreislauf: Die egozentrische Visualisierung durch AR/VR-Endgeräte und die damit verbundene multimodale Interaktion sowie die realitätsnahe Handlung der MRiLS ermöglicht ein hohes Präsenzgefühl des Nutzenden im Simulationskreislauf.

- Flexible Bereitstellung des Lerngegenstandes: Die Schulung mittels MRiLS kann standort-, endgeräte- als auch zeitunabhängig und somit entkoppelt von der realen Anlage stattfinden (z.B. vor Auslieferung der realen Anlage). Der Lerngegenstand kann dabei flexibel vervielfältigt werden, so dass jedem Lernenden ein eigener Lerngegenstand zur Verfügung steht.

- Definierbare Fehlerszenarien: Durch die Simulation des Verhaltens sowie die Anbindung an die reale Steuerungstechnik können Fehlerszenarien einfach reproduziert und ohne Gefährdung von Mensch und Maschine trainiert werden.

- Unterstützung interaktiver Lernprozesse: Die MRiLS eröffnet neue interaktive Lernprozesse und multimodale Lerneinheiten wie bspw. die maßstabsgetreue Visualisierung.

- Kopplung realer und virtueller Anlagenkomponenten: Die MRiLS ermöglicht die flexible Kopplung realer und virtueller Komponenten in einer gemeinsamen Umgebung, z.B. ein reales Bedienpanel zum Bedienen einer virtuellen Anlage in Augmented Reality.

- Integration realer Steuerungstechnik: Die MRiLS integriert bereits bestehende industrielle Steuerungstechnik sowie aus dem Engineering bereits vorhandene HiLS-Modelle, so dass keine aufwändige Nachbildung des Anlagenverhaltens für die Schulung in MR notwendig ist. Zudem kann durch diese Integration reale Hardware (z.B. ein reales Bedienpanel) flexibel in das Schulungsszenario eingebunden werden. 


\section{Anwendungsszenario}

Im Rahmen des Forschungsprojektes MRiLS wird das zuvor vorgestellte Konzept der MRiLS für das beispielhafte Automatisierungssystem "Schulungslader" der Firma Roth Steuerungstechnik GmbH umgesetzt. Um frühzeitig alle relevanten Sichtweisen bei der MRiLS zu beleuchten, wird bereits zu Beginn der technologischen Entwicklung die didaktische Perspektive auf den Lernprozess sowie die Anwenderperspektive aus Sicht der industriellen, betrieblichen sowie universitären Bildung durch die Konsortialpartner und assoziierten Partner des MRiLS-Projektes einbezogen. Die beispielhafte Implementierung dient somit neben der Validierung und Erforschung des technologischen Gesamtkonzeptes auch der Erforschung didaktischer Fragestellungen der MRiLS.

\subsection{Vorstellung Automatisierungssystem "Schulungslader"}

Das Automatisierungssystem "Schulungslader" ist ein Linienportal mit zwei translatorischen Achsen und einem pneumatischen Greifer (siehe Bild 4). Der umlaufende Materialfluss ist über eine rücklaufende Schwerkraftrollenbahn realisiert. An zwei Bearbeitungsstationen können einfache Pick\&Place Aufgaben durchgeführt werden. Das Verhalten der Bearbeitungsstationen ist über Simulationsmodelle hinterlegt.
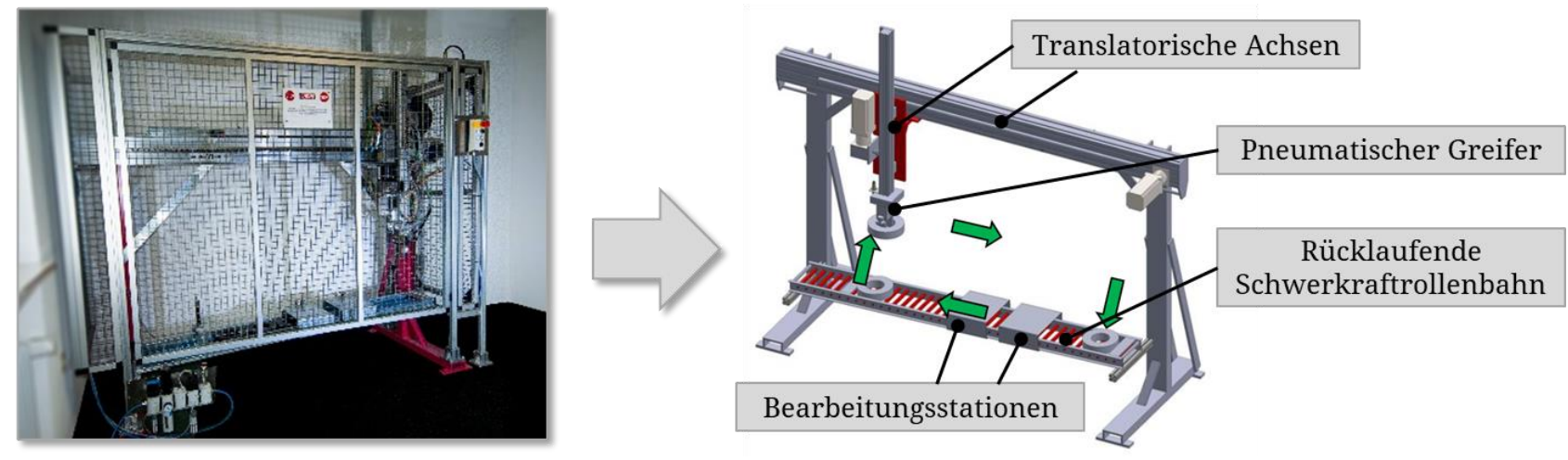

Bild 4: Aufbau des Automatisierungssystems "Schulungslader"

\subsection{Aufbau der MRiLS}

Das HiLS-Modell ist in der Echtzeit-Simulationsumgebung ISG-virtuos der Industriellen Steuerungstechnik GmbH unter Einbeziehung realer Steuerungstechnik implementiert. Die Echtzeitsimulationsumgebung ISG-virtuos ermöglicht die Simulation von Digitalen Zwillingen in Steuerungsechtzeit. Die Kopplung der Simulation mit der Steuerung erfolgt über gängige Feldbussysteme, so dass die Lösung unabhängig von Steuerungsherstellern ist.

Die Bereitstellung der MRiLS erfolgt unter Einsatz der am Virtual Automation Lab (VAL) der Hochschule Esslingen entwickelten Digital Twin as a Service Plattform (siehe auch [10]). Die Plattform agiert als Middleware (vgl. Abschnitt 2.2) und ermöglicht neben der endgeräte- und plattformunabhängigen Bereitstellung des Digitalen Zwillings auf verschiedenen Mixed Reality-Endgeräten auch die Integration verschiedener Funktionserweiterungen durch Public-Cloud-Dienste wie z.B. KI-basierte Sprachverarbeitung für multilinguale Schulungen. Über die Plattform ist sowohl ein individuelles als auch ein gemeinsames und interaktives Schulen mit dem Lernobjekt in Mixed Reality möglich. Bild 5 zeigt die Architektur der MRiLS des "Schulungsladers". 


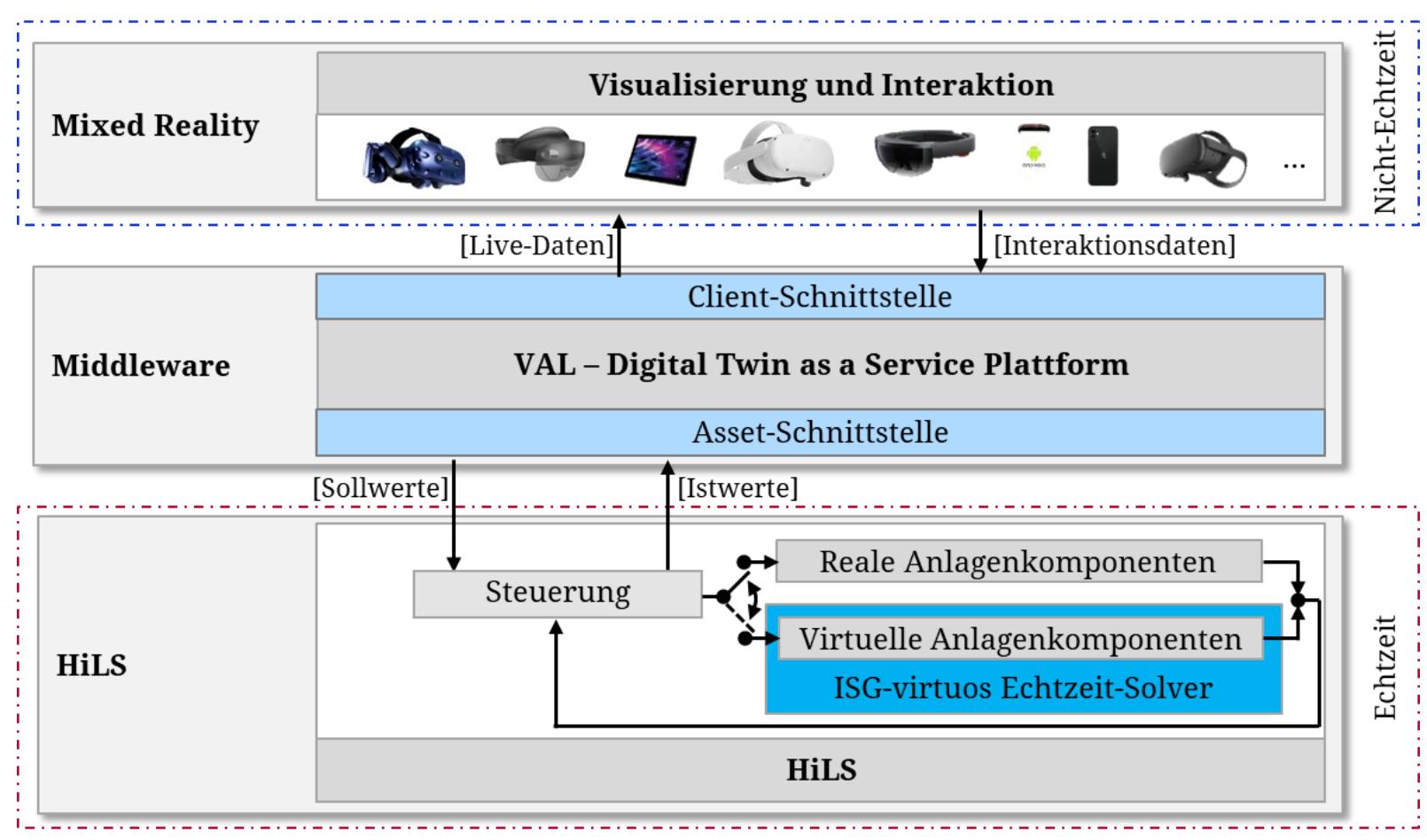

Bild 5: Architektur der MRiLS des "Schulungsladers" (vgl. [9])

Die HiLS des "Schulungsladers" umfasst neben der Implementierung des Verhaltens der virtuellen Anlagenkomponenten in ISG-virtuos auch die Anbindung an die reale Steuerungstechnik. Zur Validierung der Flexibilität des Gesamtkonzeptes wird sowohl eine Beckhoff Steuerung als auch eine Siemens Steuerung eingesetzt. Zudem kann das System mit rein virtuellen Anlagenkomponenten über das Verhaltensmodell in ISG-virtuos ohne Anbindung von realer Steuerungstechnik betrieben werden. Die industrielle Steuerungstechnik kann neben der Steuerungslogik auch die Anbindung eines realen Bedienpanels umfassen. Die HiLS koppelt sich über die Asset-Schnittstelle (bspw. über OPC UA) an die Digital Twin as a Service Plattform. Die Mixed Reality-Endgeräte verbinden sich über die ClientSchnittstelle an die Middleware und bekommen die Live-Daten für die Visualisierung übermittelt. Auf Basis dieser bereitgestellten Visualisierung können die Nutzenden über intuitive Eingabemöglichkeiten (z.B. Controller, Gestensteuerung, Sprachsteuerung) mit dem Digitalen Zwilling in Mixed Reality multimodal interagieren und dessen Verhalten manipulieren. Die Interaktionsdaten der einzelnen Nutzenden werden auf dem jeweiligen Endgerät abstrahiert und lösen entsprechende Events aus, die von jedem Endgerät direkt an die Middleware gesendet werden. In der Middleware erfolgt die Auswertung der ankommenden Events der verschiedenen Nutzenden, die Generierung von Sollwerten auf Basis dieser Events sowie die Übermittlung der Sollwerte an die angebundene HiLS. Die HiLS reagiert entsprechend dem hinterlegten Verhaltensmodell (z.B. Steuerungslogik) auf die Sollwerte und schreibt die Istwerte an die Middleware zurück. Diese Istwerte werden wiederum über die Middleware zentral an die angebundenen Endgeräte verteilt. In der Middleware existiert zu jedem Zeitpunkt ein nutzerunabhängiger konsistenter Systemzustand, so dass sich die Nutzenden flexibel während einer Schulung dazu schalten können.

Die Implementierung der MRiLS des "Schulungsladers" ermöglicht den Nutzenden, flexibel und intuitiv mit den angebundenen realen und virtuellen Anlagenkomponenten zu interagieren. Sowohl die Visualisierung als auch die Manipulation des Digitalen Zwillings sind endgeräte- (z.B. Augmented Reality-Brille, Virtual Reality-Brille, Tablet), eingabegeräte- (z.B. Controller, Datenhandschuhe) sowie ausgabegeräteübergreifend (z.B. Sprachausgabe, haptisches Feedback) auf Basis eines gemeinsamen Digitalen Zwillings durch mehrere Nutzende zeitgleich möglich.

Bild 6 zeigt einen beispielhaften Einsatz der MRiLS des "Schulungsladers" für die Schulung in Virtual Reality. 


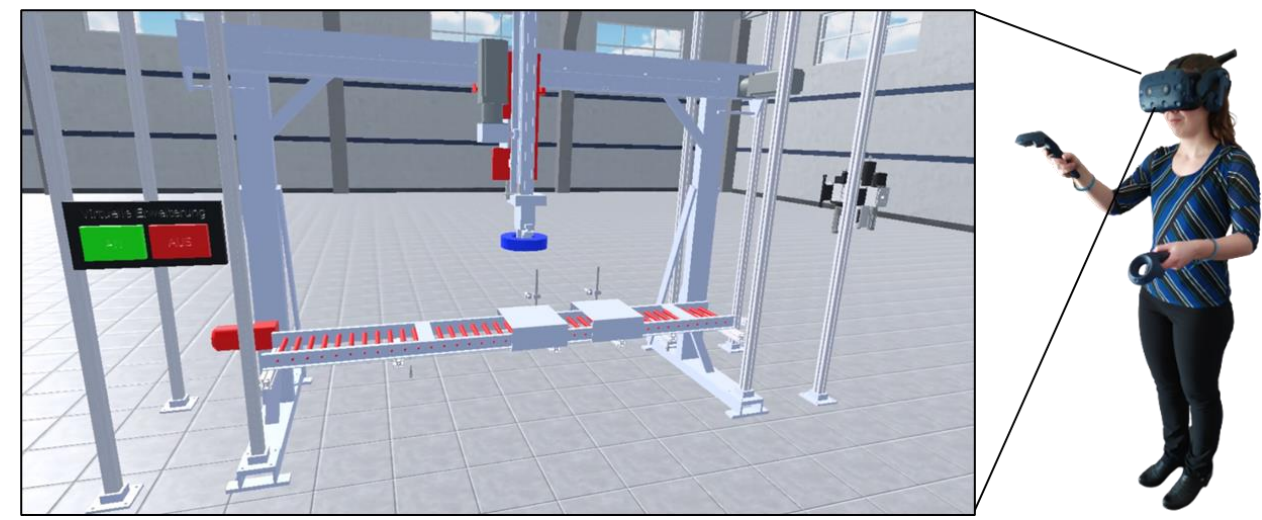

Bild 6: Beispielhafter Einsatz der MRiLS des "Schulungsladers" in Virtual Reality

\subsection{Ablauf einer Schulung}

Die Schulung mittels MRiLS gilt dabei nicht als Insellösung, sondern ist mit anderen Lehr- und Lernformen zu kombinieren und in das bestehende Schulungs-Framework zu integrieren. So kann die MRiLS beispielsweise unterstützend zum Lernen an der realen Anlage und dem Lernen mittels Bedienungsanleitung eingesetzt werden. Die MRiLS greift dabei an den Defiziten bisheriger Formen der Wissensvermittlung an und ergänzt das Schulungs-Framework um eine weitere Methode. Für die beispielhafte Implementierung des "Schulungsladers" wird die Zielgruppe der Maschinenbediener fokussiert. Bei dieser Zielgruppe ist zumeist davon auszugehen, dass die Lernenden bislang wenig Berührungspunkte beziehungsweise Erfahrungen mit Mixed Reality Technologien aufweisen. Daher kommt der Vorbereitung und Einführung der Lernenden in die virtuelle Lernumgebung durch die Schulungsleitung eine besondere Bedeutung zu. Bild 7 zeigt den geplanten Ablauf der Schulung mittels MRiLS. Nach einer kurzen Vorstellung der Schulungsanlage (z.B. durch ein Video oder an der realen Anlage), folgt eine Eingewöhnungsphase in Mixed Reality, um die Schulungsumgebung und die Bedienung kennen zu lernen und sich in der Lernumgebung zu orientieren. Das Schulungsszenario umfasst sowohl reproduktive als auch produktive selbstgesteuerte Lernphasen. In der reproduktiven Lernphase führen die Lernenden eine geleitete Bearbeitung verschiedener Aufgaben durch. Nach der Wissenseinübung und -erschließung in dieser reproduktiven Lernphase folgt die selbstgesteuerte Phase des Lernszenarios. Die Lernenden wenden dabei ihr gelerntes Wissen an und übertragen dieses beim explorativen Erkunden der Schulungsanlage auf weitere Anwendungsszenarien. Abschließend wird eine Lernzielkontrolle zur Überprüfung der Lerninhalte durchgeführt. Mögliche Wissenslücken werden durch adaptive Wiederholungen der Lerninhalte geschlossen.

\begin{tabular}{|c|c|c|c|c|}
\hline \multicolumn{5}{|c|}{ Zielgruppe: Maschinenbediener } \\
\hline Vorstellung Anlage & Eingewöhnung MR & Schulung MR & Exploration & Lernzielkontrolle \\
\hline $\begin{array}{l}\text { Kennenlernen der } \\
\text { Schulungsanlage }\end{array}$ & $\begin{array}{l}\text { Kennenlernen der } \\
\text { Schulungs- } \\
\text { umgebung in MR }\end{array}$ & $\begin{array}{l}\text { Durchführung des } \\
\text { Schulungs- } \\
\text { szenarios in MR }\end{array}$ & $\begin{array}{l}\text { Exploratives } \\
\text { Erkunden der } \\
\text { Anlage in MR }\end{array}$ & $\begin{array}{l}\text { Überprüfung der } \\
\text { Lerninhalte }\end{array}$ \\
\hline
\end{tabular}

Bild 7: Konzept des Schulungsablaufes mittels MRiLS

Für den "Schulungslader" wurden folgende relevante Schulungsszenarien für die Zielgruppe Maschinenbediener identifiziert:

- Anlage hochfahren: Der Bediener erlernt das Hochfahren der Anlage vom Einschalten des Hauptschalters bis zur Betriebsfähigkeit der Anlage.

- Not-Halt betätigen und quittieren: Der Bediener erlernt die Betätigung und das Quittieren des Not-Halts. 
- Fehlerzustände selbstständig identifizieren: Der Bediener erlernt das selbständige Erkennen von spezifischen Fehlerzuständen.

- Fehlerzustände durch manuellen Betrieb beheben: Der Bediener erlernt das manuelle Bedienen der Anlage zur Behebung der Fehlerzustände.

Die logischen Abläufe der einzelnen Schulungsszenarien sind in Flussdiagrammen hinterlegt. Bild 8 zeigt beispielhaft das Flussdiagramm für das Schulungsszenario "Not-Halt betätigen und quittieren". Die Logik umfasst sowohl die manuellen Arbeitsschritte des Lernenden als auch die hinterlegten Abfragen von Statusvariablen aus der Steuerung.

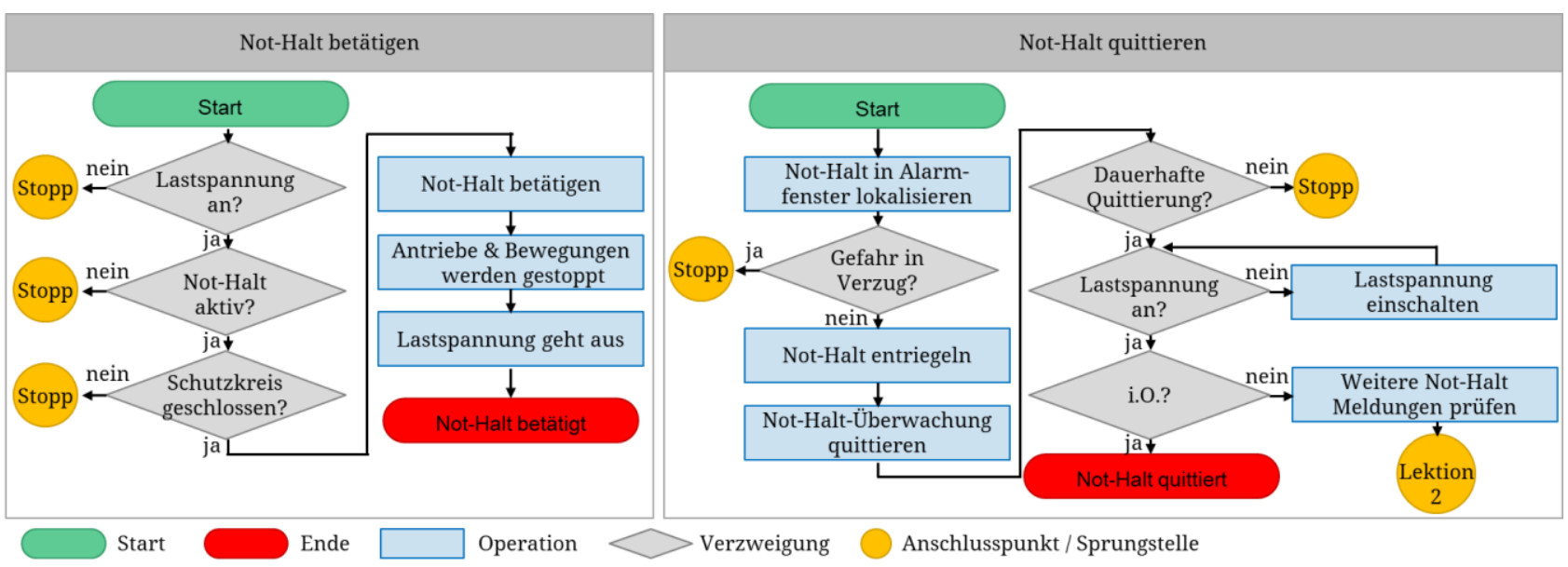

Bild 8: Flussdiagramm des Schulungsszenarios "Not-Halt betätigen und quittieren"

\section{Zusammenfassung und Ausblick}

Im Rahmen dieses Beitrages sind die bislang im Maschinen- und Anlagenbau eingesetzten Formen der Wissensvermittlung und deren Defizite aufgezeigt worden. Zur Lösung der beschriebenen Defizite wird die Mixed-Reality-in-the-Loop Simulation (MRiLS) vorgestellt. Die Funktionsfähigkeit des vorgestellten Konzeptes wird anhand eines beispielhaften Automatisierungssystems, dem "Schulungslader", validiert. Die Flexibilität und Übertragbarkeit der MRiLS wurde neben dem hier dargestellten Anwendungsbeispiel bereits bei weiteren Anwendungen, auch über die Domäne des Maschinen- und Anlagenbaus hinweg, untersucht (siehe Bild 9).

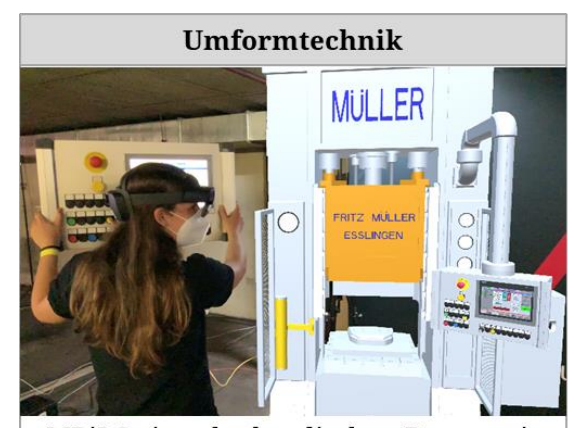

MRiLS einer hydraulischen Presse mit Anbindung an ein reales Bedienpanel

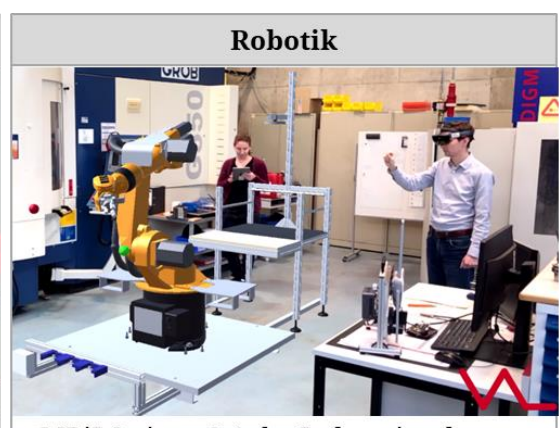

MRiLS eines 6-Achs-Industrieroboters

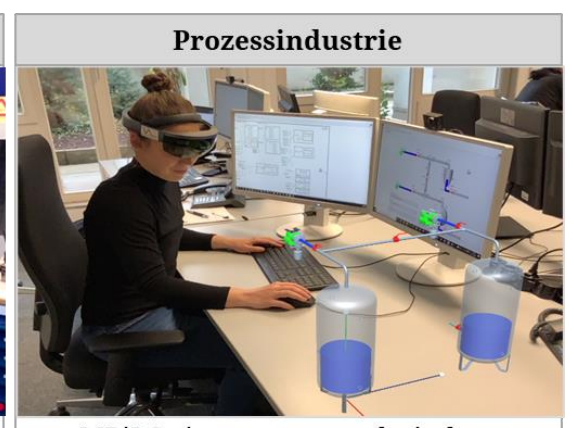

MRiLS einer prozesstechnischen Anlage mit Anbindung an LabView [11]

Bild 9: Weitere Anwendungsbeispiele der MRiLS 
In Zukunft wird das Konzept für den Ablauf der Schulung mittels MRiLS für den "Schulungslader" weiter technologisch und didaktisch untersucht sowie der praktische Einsatz in der betrieblichen, beruflichen sowie universitären Bildung erforscht. Die Schulungsmodelle der MRiLS mit den zugehörigen Flussdiagrammen sollen in Zukunft zudem automatisiert aus den Engineering-Daten einer Virtuellen Inbetriebnahme abgeleitet werden können.

\section{Danksagung}

Das diesem Bericht zugrundeliegende Vorhaben wurde mit Mitteln des Bundesministeriums für Bildung und Forschung unter den Förderkennzeichen 16SV8344 - 16 SV8348 gefördert. Die Verantwortung für den Inhalt dieser Veröffentlichung liegt bei den Autoren.

GEFÖRDERT VOM

Bundesministerium

für Bildung

und Forschung

\section{Literatur}

[1] Wehnert, C. et al.: Weiterbildung im Maschinen- und Anlagenbau mittels Mixed-Reality-in-the-Loop Simulation, Journal of Technical Education (JOTED), Bd. 9 Nr. 2, pp. 108-128, 2021.

[2] Milgram, P. et al.: Augmented Reality: A class of displays on the reality-virtuality continuum. In: Telemanipulator and Telepresence Technologies. 2351. 1994. Doi: 10.1117/12.197321.

[3] Gonzalez-Franco, M. et al.: Immersive Mixed Reality for Manufacturing Training. Frontiers in Robotics and AI, Volume 4. 2017.

[4] Shamsuzzoha, A. et al.: Digital factory - virtual reality environments for industrial training and maintenance. Interactive learning environments. 2019 doi: 10.1080/10494820.2019.1628072

[5] VDI/VDE-Gesellschaft Mess- und Automatisierungstechnik: Virtuelle Inbetriebnahme - Modellarten und Glossar, VDI/VDE 3693 Blatt 1, 2016

[6] Slater M; Wilbur, S.: A framework for immersive virtual environments (FIVE): speculations on the role of presence in virtual environments. Presence: Teleoperators and Virtual Environments, 6(6):603-616, 1997.

[7] Oh, C. S. et al.: A Systematic Review of Social Presence: Definition, Antecedents, and Implications. Frontiers in Robotics and AI, vol. 5, 2018.

[8] Hönig, J. et al.: Mixed-Reality-in-the-Loop Simulation für Schulungen im Maschinen- und Anlagenbau, Proceedings of DELFI Workshops 2021. In: Proceedings of DELFI Workshops 2021, pp. 9-22, Hochschule Ruhr West, 2021. ISBN 978-3-946757-03-0

[9] Hönig, J. et al.: Mixed-Reality-in-the-Loop Simulation von Produktionssystemen zur Aus- und Weiterbildung. atp magazin, atp 63 (6-7), Vulkan-Verlag, 2021.

[10] Schnierle, M. et al.: Mensch-Roboter-Interaktion mit Mixed Reality auf Basis einer "Digital Twin as a Service"Plattform, atp magazin 5/2019, Robotik und Digital Twin in der Smart Factory, Vulkan-Verlag, 2019.

[11] Kronberger, K.; Schnierle, M.: Mixed Reality in the Loop Simulation - Ausbildung an prozesstechnischen Anlagen auf dem Weg zum Digitalen Zwilling. In: Tagungsband Jahrestreffen der ProcessNet-Fachgemeinschaft Prozess-, Apparate- und Anlagentechnik (PAAT), 2021. 